\title{
Emergency psychiatric referrals to a university hospital in Pakistan
}

\author{
HaShim ReZA, Senior Instructor; SAJID MAHMOOD CHOUdHRY, formerly Intern; \\ and Murad Moosa Khan, Assistant Professor, Department of Psychiatry, \\ The Aga Khan University, PO Box 3500, Karachi-74800, Pakistan
}

The role of the accident and emergency department in the care of psychiatric patients has been long recognised. Mindham et al (1973) reported that many psychiatric patients, at their first or subsequent contact, present themselves as emergencies, and therefore a suitable service must be provided for them. It is interesting that a large number of reports describe various aspects of one particular service in London, namely the Maudsley Emergency Clinic. Several recent reports describe emergency psychiatric services in different district general hospitals in the United Kingdom.

These reports are not comparable because of different study designs. One major difference is that while some are based in 'walk-in' clinics, others report clinics which assessed patients referred by general practitioners; some studies report referrals from casualty departments (Ball \& Levi, 1988; Dunn \& Fernando, 1989).

The majority of referred patients were men, and emergency psychiatric assessments were mostly considered justified (Mindham et al, 1973; Ball \& Levi, 1988; Dunn \& Fernando, 1989). The diagnoses were mainly of psychoses or neuroses, with psychoses being the larger group. Schizophrenia tended to be the largest diagnostic category and there were also a substantial number of alcohol related problems.

Due to the absence of epidemiological work, knowledge of psychiatric morbidity is rudimentary in Pakistan. Akhund et al (1987) surveyed three general practices in Karachi and reported a psychiatric morbidity of $12.1 \%$, including $5.4 \%$ of anxiety-depression syndrome.

Although some teaching hospitals in Pakistan do provide emergency psychiatric assessments in their casualty departments, such a service has never been evaluated. What we know about emergency psychiatry is based on reports from the West. We therefore describe the first such service in a university teaching hospital in Pakistan.

\section{The study}

Work at the Aga Khan University Medical Centre (AKUMC), centrally located in Karachi, started in
1986. Among its various clinical services, it provides preventive and primary health care to seven squatter settlements of Karachi. The university hospital and the psychiatry service are still in the development phase. The hospital did not have a psychiatric ward until July 1990 and patients requiring in-patient psychiatric care were admitted into general medical beds. There were 19 out-patient psychiatric clinics every week with an average of 3,500 visits per annum during the study period.

Among other services offered at AKUMC is a 24 hours emergency room (ER). The ER is staffed and equipped primarily for the needs of physical emergencies. The on-call psychiatric team, comprising a resident medical officer and a consultant psychiatrist, takes referrals from the ER resident.

The aims of this study were to determine the nature of psychiatric crises which present to the emergency department of a general hospital in Pakistan and to audit the use of the psychiatric service in the ER.

The study is a retrospective case-notes survey of all the psychiatric referrals from ER between August 1986 and July 1990. The epidemiological data of the referrals, including age, sex, marital status, date and time of presenting in the ER, were documented along with the principal complaint, provisional diagnosis, management and follow-up arrangements. The diagnoses were made according to the ICD-9 criteria based on the clinical information recorded in the notes.

The statistical analyses were done on SPSS/PC+ software. The data for the male and female groups were compared for differences using the $\chi^{2}$ tests.

\section{Findings}

During the study period a total of 82,777 patients presented in ER, of whom 306 were referred on 390 occasions to the psychiatric service, giving a psychiatric morbidity rate of $0.5 \%$. The majority of patients $(53 \%)$ presented during working hours, 8 a.m. to 4 p.m., with only $9 \%$ presenting during midnight to 8 a.m.

The age of the patients ranged from 11 to 80 years with a mean of 30.39 years $($ s.d. $=13.01)$. Women 
$(63 \%)$ were relatively younger (mean age 29.17 years, s.d. $=12.41$ ) than men (mean age 32.49 years, s.d. = $13.80)$. The majority of both women $(61 \%)$ and men (54\%) were married.

Pain was the principal presenting complaint in $23 \%$, with other physical complaints in another $37 \%$. Low mood, agitation, bizarre behaviour and violence towards self or others were the chief psychological complaints in $39 \%$.

The largest diagnostic group (42\%) was that of affective disorders. Other diagnoses were neuroses $(22 \%)$, schizophrenia and paranoid states $(8 \%)$, organic psychoses $(5 \%)$, other psychiatric illnesses $(16 \%)$ and no psychiatric disorder $(3 \%)$. There were only two cases of alcohol related problems and none of a personality disorder.

Management in the ER resulted in discharge for $6 \%$, referral to other services for $11 \%$, admission into a psychiatric bed for $13 \%$ and out-patient follow-up for $69 \%$. Only $18 \%$ were regular in their follow-up; $43 \%$ never attended the follow-up clinic. A significant number (17\%) made 84 repeat visits to ER after the index visit.

In all the variables women and married persons predominated but did not reach statistical significance except for provisional diagnoses $\left(\chi^{2}=27.58\right.$, d.f. $=4, P=<0.001$ ) and follow-up compliance $\left(\chi^{2}=10.62\right.$, d.f. $\left.=4, P=0.031\right)$.

\section{Comment}

There was an increase of emergency psychiatric referrals over the years, which probably reflects the increasing awareness of the availability of services in AKUMC and does not necessarily suggest an increasing acceptance of psychiatric illness. The psychiatric morbidity rate of $0.5 \%$, considerably smaller than comparable figures in British casualty departments and Karachi general practices, is an underestimate. The referrals were made by the ER resident and were influenced by his psychiatric knowledge - in fact more than $93 \%$ of the referrals were diagnosed as having a psychiatric disorder. However, many cases probably went unnoticed.

More than $60 \%$ of our patients presented with physical complaints although the majority of them were later diagnosed as suffering from a mood disorder. This supports the notion that people in industrially developing societies present with somatic rather than psychological complaints when diagnosed as suffering from psychiatric illnesses. The large number of affective disorders also suggests a difference in the clinical work of the emergency psychiatric service in AKUMC compared with the services reported in the literature.

The large number of married women in our sample also differs from the results reported in the literature. Marriage, instead of protecting against psychiatric morbidity, is a significant risk factor for women in Pakistani culture. Naeem (1990) mentioned low selfesteem, early marriage, hostile in-laws and lack of a confiding relationship with the husband as important social factors associated with depression in Pakistani women. The absence of a comprehensive primary health care system may also explain why relatively few severe psychiatric illnesses, especially in a group of married women, presented in the ER. In good primary health care systems women of reproductive age contact their GPs more often than men. This provides an opportunity for their psychiatric problems to be noticed before reaching crisis point. Such care could have obviated the need for emergency psychiatric assessment in many of our referrals.

In conclusion, our study shows important differences to those reported in the Western literature: emergency psychiatric referrals presented mostly during the day, there was a preponderance of women and married persons, and affective disorders were the largest diagnostic group. On the other hand, the mean age, the low rate of those requiring in-patient treatment and poor compliance with out-patient follow-up are similar to the trends reported.

\section{Acknowledgements}

We would like to thank Drs S. Hussain and A. Faizi for allowing access to their case-notes and $\mathrm{Mr} \mathrm{Z}$. R. Meghani for his untiring help throughout this project.

\section{References}

Akhund, F., Khan, M. F., Mohsin, A. \& Ahmed, S. H. (1987) Psychiatric disorders in general practice. Journal of the Pakistan Medical Association, 37, 2-4.

BALL, H. N. \& LEVI, M. I. (1988) A casualty psychiatric clinic at the Royal Liverpool Hospital. Bulletin of the Royal College of Psychiatrists, 12, 333-334.

DUNN, J. \& FERNANDO, R. (1989) Psychiatric presentations to an accident and emergency department. Psychiatric Bulletin, 13, 672-674.

MindhaM, R. H. S., Kelleher, M. J. \& BiRley, J. L. T. (1973) A psychiatric casualty department. Lancet, 1, 1169-1171.

NaEem, S. (1990) Psycho-social risk factors for depression in Pakistani women. Academic dissertation for FCPS, College of Physicians and Surgeons of Pakistan. 